\title{
Genetic testing for Marfan-like disorders
}

\author{
Yeltay Rakhmanov ${ }^{1}$, Paolo Enrico Maltese ${ }^{1 *}$, Stefano Paolacci ${ }^{2}$, Carla Marinelli ${ }^{1}$, Marco Castori ${ }^{3}$, \\ Tommaso Beccari ${ }^{4}$, Munis Dundar ${ }^{5}$ and Matteo Bertelli ${ }^{1,2}$
}

\begin{abstract}
Marfan-like disorders are inherited conditions with features resembling Marfan syndrome but without a pathogenic variant in FBN1, and/or without a clinical diagnosis of Marfan syndrome according to the Revised Ghent criteria, and/or with a pathogenic variant in a different disease gene. Marfan-like disorders are clinically and genetically heterogeneous and have variable prognosis. They may have autosomal dominant or autosomal recessive patterns of inheritance. The prevalence of most Marfan-like disorders is unknown. This Utility Gene Test was prepared on the basis of an analysis of the literature and existing diagnostic protocols. Molecular testing is useful for diagnosis confirmation, as well as differential diagnosis, appropriate genetic counselling and access to clinical trials.
\end{abstract}

Keywords: Marfan-like syndromes, Loeys-Dietz syndromes, familial thoracic aortic aneurism, cutis laxa syndromes, bicuspid aortic valve disease, arterial tortuosity syndrome. EBTNA UTILITY GENE TEST

'MAGI's Lab, Rovereto, Italy

${ }^{2}$ MAGI Euregio, Bolzano, Italy

${ }^{3}$ Division of Medical Genetics, IRCCS-Casa Sollievo della Sofferenza, San Giovanni Rotondo (FG), Italy

${ }^{4}$ Department of Pharmaceutical Sciences, University of Perugia, Perugia, Italy

${ }^{5}$ Department of Medical Genetics, Erciyes University Medical School, Kayseri, Turkey

*Corresponding author: P. E. Maltese E-mail: paolo.maltese@assomagi.org

DOI: 10.2478/ebtj-2018-0033
(C) 2018 Authors. This work was licensed under the Creative Commons AttributionNonCommercial-NoDerivs 3.0 License.

\section{Marfan-like disorders}

(Other synonyms: Loeys-Dietz syndromes, arterial tortuosity syndrome, thoracic aortic aneurysms and dissections, thoracic aortic aneurysms and dissections associated with bicuspid aortic valve, aortic aneurysms with cutis laxa)

\section{General information about the disease}

Marfan-like disorders are inherited conditions with features resembling Marfan syndrome but that do not match all the diagnostic criteria of the Revised Ghent Nosology (2010) and/or that do not share a pathogenic variant in FBN1. Marfan-like disorders group together different systemic connective tissue disorders caused by mutations in various genes and have different clinical manifestations. For example, Loeys-Dietz syndromes appear with aortic aneurysms and dissections, widespread arterial tortuosity/aneurysms, bifid uvula and hypertelorism (wide spaced eyes) (1). Arterial tortuosity syndrome is mainly characterized by tortuosity and elongation of the large- and medium-sized arteries with a predisposition for stenosis and aneurysms (2). In non-syndromic thoracic aortic aneurysms and dissections, the most common site of aneurysms is the infrarenal abdominal aorta, followed by the ascending thoracic aorta (3). Cutis laxa (loose skin) may have variable systemic involvement (inguinal hernia, cardiopulmonary disease and emphysema) (4).

The prevalence of Marfan-like disorders is unknown.

To establish a clinical diagnosis or suspicion, the following multiple diagnostic procedures should be used: clinical examination and history, heart ultrasound, eye examination with slit lamp for lens dislocation, advanced imaging studies of the vascular tree. 
Differential diagnosis should consider Marfan syndrome, congenital contractural arachnodactyly/Beals syndrome, homocystinuria caused by cystathionine $\beta$-synthase deficiency, Stickler syndrome and fragile $\mathrm{X}$ syndrome.

Marfan-like disorders have different phenotypes and clinical features. Inheritance is usually autosomal dominant; cutis laxa, Ehlers-Danlos syndromes and moyamoya disease may show autosomal recessive or dominant transmission patterns (2-11).

\section{Autosomal dominant Marfan-like syndromes}

- MASS syndrome (OMIM disease 604308) - FBN1 (OMIM gene 134797);

- Shprintzen-Goldberg craniosynostosis syndrome (SGS, OMIM disease 182212) - SKI (OMIM gene 164780);

- Loeys-Dietz syndrome 1 (LDS1, OMIM disease 609192) TGFBR1 (OMIM gene 190181);

- Loeys-Dietz syndrome 2 (LDS2, OMIM disease 610168) TGFBR2 (OMIM gene 190182);

- Loeys-Dietz syndrome 3 (LDS3, OMIM disease 613795) SMAD3 (OMIM gene 603109);

- Loeys-Dietz syndrome 4 (LDS4, OMIM disease 614816) TGFB2 (OMIM gene 190220);

- Loeys-Dietz syndrome 5 (LDS5, OMIM disease 615582) TGFB3 (OMIM gene 190230);

- Familial thoracic aortic aneurysm 4 (AAT4, OMIM disease 132900) - MYH11 (OMIM gene 160745);

- Familial thoracic aortic aneurysm 6 (AAT6, OMIM disease 611788) - ACTA2 (OMIM gene 102620);

- Familial thoracic aortic aneurysm 7 (AAT7, OMIM disease 613780) - MYLK (OMIM gene 600922);

- Familial thoracic aortic aneurysm 8 (AAT8, OMIM disease 615436) - PRKG1 (OMIM gene 176894);

- Familial thoracic aortic aneurysm 9 (AAT9, OMIM disease 616166) - MFAP5 (OMIM gene 601103);

- Familial thoracic aortic aneurysm 10 (AAT10, OMIM disease 617168) - LOX (OMIM gene 153455);

- Cutis laxa autosomal dominant 1 (ADCL1, OMIM disease 123700) - ELN (OMIM gene 130160);

- Cutis laxa autosomal dominant 2 (ADCL2, OMIM disease 614434) - FBLN5 (OMIM gene 604580);

- Cutis laxa autosomal dominant 3 (ADCL3, OMIM disease 616603) - ALDH18A1 (OMIM gene 138250);

- Aortic valve disease 1 (AOVD1, OMIM disease 109730) NOTCH1 (OMIM gene 190198);

- Aortic valve disease 2 (AOVD 2, OMIM disease 614823) SMAD6 (OMIM gene 602931);

- Moyamoya disease 5 (MYMY5, OMIM disease 614042) ACTA2 (OMIM gene 102620);

- Ehlers-Danlos syndrome, classic type 1 (EDSCL1, OMIM disease 130000) - COL5A1 (OMIM gene 120215);

- Ehlers-Danlos syndrome, classic type 2 (EDSCL2, OMIM disease 130010) - COL5A2 (OMIM gene 120190);

- Ehlers-Danlos syndrome, periodontal type 1 (EDSPD1, OMIM disease 130080) - C1R (OMIM gene 613785);

- Ehlers-Danlos syndrome, periodontal type 2 (EDSPD2,
OMIM disease 617174) - C1S (OMIM gene 120580);

- Ehlers-Danlos syndrome, arthrochalasia type (EDSARTH, OMIM disease 130060) - COL1A1 (OMIM gene 120150);

- Ehlers-Danlos syndrome, vascular type (EDSVASC, OMIM disease 130050) - COL3A1 (OMIM gene 120180).

\section{Autosomal recessive Marfan-like syndromes}

- Arterial tortuosity syndrome (ATS, OMIM disease 208050) SLC2A10 (OMIM gene 606145);

- Autosomal recessive cutis laxa type 1A (ARCL1A, OMIM disease 219100) - FBLN5 (OMIM gene 604580);

- Autosomal recessive cutis laxa type 1B (ARCL1B, OMIM disease 614437) - EFEMP2 (OMIM gene 604633);

- Autosomal recessive cutis laxa type 2A (ARCL2A, OMIM disease 219200) - ATP6V0A2 (OMIM gene 611716);

- Autosomal recessive cutis laxa type 3A (ARCL3A, OMIM disease 219150) - ALDH18A1 (OMIM gene 138250);

- Moyamoya disease 6 with achalasia (MYMY6, OMIM disease 615750) - GUCY1A3 (OMIM gene 139396);

- Ehlers-Danlos syndrome, dermatosparaxis type(EDSDERMS, OMIM disease 225410) - ADAMTS2 (OMIM gene 604539);

- Ehlers-Danlos syndrome, kyphoscoliotic type 1 (OMIM disease 225400) - PLOD1 (OMIM gene 153454);

- Ehlers-Danlos syndrome, kyphoscoliotic type 2 (EDSKSCL2, OMIM disease 614557) - FKBP14 (OMIM gene 614505);

- Ehlers-Danlos syndrome, spondylodysplastic type 1 (EDSSPD1, OMIM disease 130070) - B4GALT7 (OMIM gene 604327);

- Ehlers-Danlos syndrome, spondylodysplastic type 2 (EDSSPD2, OMIM disease 615349) - B3GALT6 (OMIM gene 615291);

- Ehlers-Danlos syndrome, spondylodysplastic type 3 (EDSSPD3, OMIM disease 612350) - SLC39A13 (OMIM gene 608735);

- Ehlers-Danlos syndrome, cardiac valvular type (EDSCV, OMIM disease 225320) - COL1A2 (OMIM gene 120160);

- Ehlers-Danlos syndrome, musculocontractural type 1 (EDSMC1, OMIM disease 601776) - (CHST14 OMIM gene 608429);

- Ehlers-Danlos syndrome, musculocontractural type 2 (EDSMC2, OMIM disease 615539) - DSE (OMIM gene 605942);

- Ehlers-Danlos syndrome, classic-like (EDSCLL, OMIM disease 606408) - TNXB (OMIM gene 600985).

Pathogenic variants may include missense, nonsense, splicing, small insertions and deletions, small indels, gross insertions, duplications.

\section{Aims of the test}

- To determine the gene defect responsible for the disease;

- To confirm clinical diagnosis;

- To assess the recurrence risk and perform genetic counselling for at-risk/affected individuals. 


\section{Test characteristics}

\section{Specialist centers/ Published Guidelines}

The test is listed in the Orphanet database and is offered by $47 \mathrm{ac}-$ credited medical genetic laboratories in the EU, and in the GTR database, offered by 14 accredited medical genetic laboratories in the US.

Guidelines for clinical use of the test are described in Genetics Home Reference (ghr.nlm.nih.gov) and Gene Reviews (5).

\section{Test strategy}

A multi-gene next generation sequencing panel is used for the detection of nucleotide variations in coding exons and flanking introns of the above genes. Potentially causative variants and regions with low coverage are Sanger-sequenced. Sanger sequencing is also used for family segregation studies.

Multiplex Ligation Probe Amplification (MLPA) is used to detect duplications and deletions in TGFBR1 and TGFBR2.

To perform molecular diagnosis, a single sample of biological material is normally sufficient. This may be $1 \mathrm{ml}$ peripheral blood in a sterile tube with $0.5 \mathrm{ml} \mathrm{K}$ EDTA or $1 \mathrm{ml}$ saliva in a sterile tube with $0.5 \mathrm{ml}$ ethanol $95 \%$. Sampling rarely has to be repeated. Gene-disease associations and the interpretation of genetic variants are rapidly developing fields. It is therefore possible that the genes mentioned in this note may change as new scientific data is acquired. It is also possible that genetic variants today defined as of "unknown or uncertain significance" may acquire clinical importance.

\section{Genetic test results}

\section{Positive}

Identification of pathogenic variants in the above genes confirms the clinical diagnosis and is an indication for family studies.

A pathogenic variant is known to be causative for a given genetic disorder based on previous reports, or predicted to be causative based on loss of protein function or expected significant damage to proteins or protein/protein interactions. In this way it is possible to obtain a molecular diagnosis in new/other subjects, establish the risk of recurrence in family members and plan preventive and/or therapeutic measures.

\section{Inconclusive}

Detection of a variant of unknown or uncertain significance (VUS): a new variation without any evident pathogenic significance or a known variation with insufficient evidence (or with conflicting evidence) to indicate it is likely benign or likely pathogenic for a given genetic disorder. In these cases, it is advisable to extend testing to the patient's relatives to assess variant segregation and clarify its contribution. In some cases, it could be necessary to perform further examinations/tests or to do a clinical reassessment of pathological signs.

\section{Negative}

The absence of variations in the genomic regions investigated does not exclude a clinical diagnosis but suggests the possibility of:

- alterations that cannot be identified by sequencing, such as large rearrangements that cause loss (deletion) or gain (duplication) of extended gene fragments;

- sequence variations in gene regions not investigated by this test, such as regulatory regions ( $5^{\prime}$ and $3^{\prime}$ UTR) and deep intronic regions;

- variations in other genes not investigated by the present test.

\section{Unexpected}

Unexpected results may emerge from the test, for example information regarding consanguinity, absence of family correlation or other genetically based diseases.

\section{Risk for progeny}

If the identified pathogenic variant has autosomal dominant transmission, the probability that an affected carrier transmit the disease variant to his/her children is $50 \%$ in any pregnancy, irrespective of the sex of the child conceived.

In autosomal recessive mutations, both parents are usually healthy carriers. In this case, the probability of transmitting the disorder to the offspring is $25 \%$ in any pregnancy of the couple, irrespective of the sex of the child. An affected individual generates healthy carrier sons and daughters in all cases, except in pregnancies with a healthy carrier partner. In these cases, the risk of an affected son or daughter is $50 \%$.

\section{Limits of the test}

The test is limited by current scientific knowledge regarding the gene and disease.

Analytical sensitivity (proportion of positive tests when the genotype is truly present) and specificity (proportion of negative tests when the genotype is not present)

NGS Analytical sensitivity $>99.99 \%$, with a minimum coverage of 10X; Analytical specificity $99.99 \%$.

SANGER Analytical sensitivity $>99.99 \%$; Analytical specificity 99.99\%.

MLPA Analytical sensitivity $>99.99 \%$; Analytical specificity 99.99\%.

Clinical sensitivity (proportion of positive tests if the disease is present) and clinical specificity (proportion of negative tests if the disease is not present)

Clinical sensitivity: variations in the aforementioned genes are linked to Marfan-like syndromes, but may be individual variations (identified in one or a few families) and total epidemiological data is therefore not available.

Clinical specificity: data not available.

\section{Prescription appropriateness}

The genetic test is appropriate when:

a) the patient meets the diagnostic criteria for a Marfan-like syndrome; 
b) the sensitivity of the test is greater than or equal to that of tests described in the literature.

\section{Clinical utility}

\section{Clinical management}

Confirmation of clinical diagnosis Utility

Differential diagnosis Yes

Couple risk assessment Yes Yes

Availability of clinical trials can be checked on-line at https://clinicaltrials.gov/

\section{References}

1. Loeys BL, Chen J, Neptune ER, Judge DP, Podowski M, Holm T, Meyers J, Leitch CC, Katsanis N, Sharifi N, Xu FL, Myers LA, Spevak PJ, Cameron DE, De Backer J, Hellemans J, Chen Y, Davis EC, Webb CL, Kress W, Coucke P, Rifkin DB, De Paepe AM, Dietz HC. A syndrome of altered cardiovascular, craniofacial, neurocognitive and skeletal development caused by mutations in TGFBR1 or TGFBR2. Nat Genet 2005; 37(3): 275e81.

2. Castori M, Ritelli M, Zoppi N, Molisso L, Chiarelli N, Zaccagna F, Grammatico P, Colombi M. Adult presentation of arterial tortuosity syndrome in a 51-year-old woman with a novel homozygous c. $1411+1 \mathrm{G}>$ A mutation in the SLC2A10 gene. Am J Med Genet A 2012; 158A:1164-69.

3. Pannu H, Avidan N, Tran-Fadulu V, Milewicz DM. Genetic basis of thoracic aortic aneurysms and dissections: potential relevance to abdominal aortic aneurysms. Ann N Y Acad Sci 2006; 1085: 24255.
4. Hbibi, M., Abourazzak, S., Idrissi, M., Chaouki, S., Atmani, S., \& Hida, M. Cutis Laxa syndrome: a case report. Pan Afr Med J 2015; 20: 3.

5. Loeys BL, Dietz HC. Loeys-Dietz Syndrome. In: MP Adam, HH Ardinger, RA Pagon, SE Wallace, LJH Bean, et al., editors. GeneReviews(R) 1993; Seattle (WA).

6. Malfait F, Wenstrup R, De Paepe A. Ehlers-Danlos syndrome, classic type. In: MP Adam, HH Ardinger, RA Pagon, SE Wallace, LJH Bean, et al., editors. GeneReviews 2007; Seattle (WA).

7. Pepin MG, Murray ML, Byers PH. Vascular Ehlers-Danlos syndrome. In: MP Adam, HH Ardinger, RA Pagon, SE Wallace, LJH Bean, et al., editors. GeneReviews 1999; Seattle (WA).

8. Yeowell HN, Steinmann B. Ehlers-Danlos syndrome, kyphoscoliotic form. In: MP Adam, HH Ardinger, RA Pagon, SE Wallace, LJH Bean, et al., editors. GeneReviews 2000; Seattle (WA).

9. Levy HP. Ehlers-Danlos syndrome, hypermobility type. In: MP Adam, HH Ardinger, RA Pagon, SE Wallace, LJH Bean, et al., editors. GeneReviews 2004; Seattle (WA).

10. Guo DC, Papke CL, Tran-Fadulu V, Regalado ES, Avidan N, Johnson RJ, Kim DH, Pannu H, Willing MC, Sparks E, Pyeritz RE, Singh MN, Dalman RL, Grotta JC, Marian AJ, Boerwinkle EA, Frazier LQ, LeMaire SA, Coselli JS, Estrera AL, Safi HJ, Veeraraghavan S, Muzny DM, Wheeler DA, Willerson JT, Yu RK, Shete SS, Scherer SE, Raman CS, Buja LM, Milewicz DM. Mutations in smooth muscle alpha-actin (ACTA2) cause coronary artery disease, stroke, and Moyamoya disease, along with thoracic aortic disease. Am J Hum Genet 2009; 84(5):617-27.

11. Hervé D, Philippi A, Belbouab R, Zerah M, Chabrier S, Collardeau-Frachon S, Bergametti F, Essongue A, Berrou E, Krivosic V, Sainte-Rose C, Houdart E, Adam F, Billiemaz K, Lebret M, Roman S, Passemard S, Boulday G, Delaforge A, Guey S, Dray X, Chabriat $H$, Brouckaert $P$, Bryckaert M, Tournier-Lasserve E. Loss of a $1 \beta 1$ soluble guanylate cyclase, the major nitric oxide receptor, leads to moyamoya and achalasia. Am J Hum Genet 2014; 94(3):385-94. 